\title{
Topiramate in the prevention of pediatric migraine: literature review
}

\author{
Diana Ferraro · Girolamo Di Trapani
}

Received: 27 November 2007 / Revised: 7 March 2008/Accepted: 10 March 2008/Published online: 2 April 2008

(C) Springer-Verlag 2008

\begin{abstract}
Pediatric migraine is a disabling condition, which can cause a significant impact on quality of life. Currently, no drugs have been approved by the FDA for its preventive treatment. Our aim was to review the medical literature concerning the efficacy and tolerability of topiramate in the prophylactic treatment of migraine in children and adolescents. A total of five papers were reviewed: two randomized controlled trials (RCTs), a posthoc subset analysis of adolescents who had been included in three RCTs carried out on adults and two open studies. Topiramate has been proven to reduce headache frequency and the accompanying disability. The frequency of side effects varied considerably among studies, the most frequent being weight loss, anorexia, abdominal pain, difficulties in concentrating, sedation and paresthesia. Since these adverse events, although often transitory, may be distressing for the child, we strongly recommend to assess the disability caused by the migraine episodes before deciding to initiate a prophylactic treatment. Nevertheless, dropout rates due to side effects in the studies were very low.
\end{abstract}

Keywords Migraine $\cdot$ Headache $\cdot$ Children - Pediatric . Topiramate

D. Ferraro · G. Di Trapani $(\bowtie)$

Headache Center, Neurology Institute, Policlinico A. Gemelli, Università Cattolica del Sacro Cuore, Largo A. Gemelli, 8, 00168 Rome, Italy

e-mail: gditrapani@rm.unicatt.it

D. Ferraro

e-mail: perdiana@tin.it

\section{Introduction}

Pediatric migraine is a disabling condition, which can cause a significant impact on quality of life. It can negatively influence the child's school performances, social and family life and often causes school absenteeism. Migraine prevalence in childhood ranges from 2.7 to $10 \%$ [1]. About $3-5 \%$ of school-aged children suffer from migraine and this proportion gradually increases to $20 \%$ through adolescence. Initially, there is a slight male predominance; however, through adolescence, there is a shift toward female predominance, which remains through adulthood $[2,3]$.

Management of pediatric migraine includes lifestyle changes (in order to avoid foods, habits or enviromental factors that may trigger a migraine attack), the use of abortive medications and preventive measures, which can be either nonpharmacological or pharmacological. Guidelines addressing the indications for the use of preventive measures in children are lacking, but to date, most authors recommend preventive measures in children when headache frequency exceeds three to four episodes per month and/or the attacks are significantly disabling, as measured in scoring systems such as the Pediatric Migraine Disability Assessment Scale (PedMIDAS) [4, 5]. PedMIDAS is a validated six-item questionnaire based on the adult MIDAS tool [6] with developmentally appropriate changes and adjustments for childhood lifestyle. The questions deal with the impact of headache on school (school day absences, partial day absences, functioning at $50 \%$ or less ability in school), on household acitivities (ability to perform homework and chores) and on social functioning (including sports) [7, 8]. The assessment of migraine disability is particularly important in children to correctly evaluate and treat migraine. 
Currently, no drugs have been approved by the Food and Drug Administration (FDA) for the preventive treatment of pediatric migraine. Topiramate is an antiepileptic drug, which has been approved by the FDA in the United States and in many other countries for the preventive treatment of migraine in adults and for the treatment of partial-onset seizures and primary generalized tonic-clonic seizures as add-on therapy in children as young as 2 years. Our aim was to review the medical literature concerning the efficacy and tolerability of topiramate in the prophylactic treatment of migraine in children and adolescents.

\section{Materials and methods}

We searched Pubmed (1966-July 2007) using the following keywords: "topiramate" + "headache" or "migraine" + "children" or "adolescents" or "pediatric." We included randomized controlled trials (RCT), open-label trials and retrospective studies. We excluded case-reports and studies conducted on less than 10 patients. We classified the studies in accordance with the AAN classification of evidence for therapeutic intervention (Table 1) [9]. A total of five papers were reviewed.

\section{Results}

We found two RCTs, a post-hoc subset analysis of 51 adolescents (12-17 years) who had been enrolled in three

Table 1 AAN classification of evidence for therapeutic intervention

Class I: Prospective, randomized, controlled clinical trial with masked outcome assessment, in a representative population.

The following are required:

(a) Primary outcome(s) is/are clearly defined

(b) Exclusion/inclusion criteria are clearly defined

(c) Adequate accounting for drop-outs and crossovers with numbers sufficiently low to have minimal potential for bias

(d) Relevant baseline characteristics are presented and substantially equivalent among treatment groups or there is appropriate statistical adjustment for differences

Class II: Prospective matched group cohort study in a representative population with masked outcome assessment that meets (a)-(d) above OR a RCT in a representative population that lacks one criteria (a)-(d)

Class III: All other controlled trials (including well-defined natural history controls or patients serving as own controls) in a representative population, where outcome is independently assessed, or independently derived by objective outcome measurement.

Class IV: Evidence from uncontrolled studies, case series, case reports, or expert opinion. pivotal RCTs of topiramate for migraine prophylaxis in adults and two open studies.

In a recent Class I randomized, double-blind, placebocontrolled trial [10], 44 children were randomized (in a 1:1 ratio) to receive either placebo or topiramate (titrated to $100 \mathrm{mg}$ a day) for 12 weeks. Participants had a diagnosis of migraine without aura (according to 2004 International Headache Society criteria) [11] and a frequency of two or more headaches per month for 3 months before entering the study. Children with comorbid medical associations or who were already on migraine prophylaxis were excluded. Primary outcome measures were the reduction in migraine frequency and severity; secondary outcome measures included number of analgesics taken and the functional disability. Migraine monthly frequency decreased from $16.14( \pm 9.35)$ at baseline to $4.27( \pm 1.95)$ at the end of the study in the treated group as compared with a decrease from $13.38( \pm 7.78)$ to $7.48( \pm 5.94)$ in the placebo group $(P=0.025)$. The percentage of topiramate-treated subjects showing $>50 \%$ reduction in monthly migraine days during the double-blind phase was $95.2 \%$ as opposed to $52.4 \%$ in the placebo group $(P=0.002)$. The Pediatric Migraine Disability Assessment Score (PedMIDAS) also decreased significantly $(P=0.003)$ as did school absenteeism $(P=0.002)$. Side effects were rated mild to moderate, did not interfere with daily activities or cause any dropouts and included weight-loss (mean loss of $0.3 \mathrm{~kg}$ ) in $81 \%$ of patients, paresthesia (23.8\%), loss of appetite (23.8\%), lack of concentration (19\%), sedation (19\%) and abdominal pain $(14.3 \%)$. This seems a well-performed trial in which the mean monthly migraine frequency reduction is clearly evident in the topiramate-treated group. Patients treated with topiramate suffered at least five episodes a month before entering the study (range 5-30); a preventive treatment was, thus, clearly indicated. The reduction in the PedMIDAS score indicates that the drug side effects (difficulties in concentrating, sedation) interfered with school, household or social activities to a lesser extent than the migraine episodes.

The other Class I, randomized, double-blind, placebocontrolled trial [12] was carried out on 162 children (age 615 years) who were randomized in a $2: 1$ ratio to receive either topiramate (which was titrated over 8 weeks to 2$3 \mathrm{mg} / \mathrm{kg}$ per day and maintained for 12 weeks) or placebo. Eligible criteria were the children with migraine with or without aura, weighing more than $20 \mathrm{~kg}$, who experienced 3-10 migraine days/month for the 3 months prior to the screening and during the 4-week prospective baseline phase. Exclusion criteria included chronic migraine, analgesic overuse and previous failure of $\geq 2$ adequately dosed migraine preventive medications. One hundred and fiftyseven subjects were included in the intention to treat (ITT) population, defined as randomized subjects who had 
received at least one dose of study medication and had at least one postbaseline efficacy assessment, and a total of 131 children completed the study (per-protocol population); of these, 108 were treated with topiramate. Primary outcome measure was the reduction of migraine days per month in each treatment group of the ITT population. During the double-blind-phase, relative to the 4-week prospective baseline phase, there was a reduction of $2.6 \pm 2.6$ migraine days per month in the topiramate group compared with a mean reduction of $2.0 \pm 3.1$ migraine days per month in the placebo group $(P=0.061)$. In the per-protocol population, the reduction in monthly migraine days $(2.8 \pm 2.4$ as opposed to $2.2 \pm 2.1$ in the placebo group) reached statistical significance $(P=0.033)$. During the last 28 days of treatment, the mean number of monthly migraine was reduced by $3.1 \pm 2.6$, as opposed to $2.4 \pm 2.8$ in the placebo group $(P=0.023)$. In the treated group, $32 \%$ of patients experienced a $\geq 75 \%$ reduction in mean monthly migraine days compared with a $14 \%$ reduction in the placebo group $(P=0.02)$. The dropout rate for adverse events was $6.3 \%$ for the topiramate group and $4 \%$ for the placebo group. In the treated group, the most common adverse events were anorexia (13\%); weight decrease $(10.2 \%$ ), with a mean loss of $1.4 \pm 2.6 \mathrm{~kg}$ (as opposed to $0.7 \pm 3.9 \mathrm{~kg}$ in the placebo group); abdominal pain $(10.2 \%)$; paresthesia (8.3\%); and somnolence $(8.3 \%)$. Serious adverse events occurred in four topiramate-treated patients and included infection $(n=2)$, severe migraine $(n=1)$ and suicidal ideation $(n=1)$.

In this study, the mean monthly migraine frequency reduction seems less evident when compared to the previous study [10], probably because migraine frequency at baseline was lower (range 2-9 in the ITT topiramate population) and children with chronic migraine were excluded. An important aspect is that the reduction is more evident in the last 28 days of treatment, indicating that it may take a few weeks before treatment with topiramate reaches its maximum efficacy.

Winner et al. [13] performed a post-hoc subset analysis on 51 adolescents (12-17 years) who had been enrolled in three pivotal trials of topiramate for migraine prophylaxis in adults. Patients were to have had between 3 and 12 migraine attacks, and no more than 14 headache days per 28 days during the 3 months prior to the screening and during the 4-week prospective baseline phase. Also excluded were patients who overused analgesics or who had failed to respond to two or more adequate prophylactic treatments. Topiramate was administered at the dosages of 50,100 or $200 \mathrm{mg}$ a day for 26 weeks. When compared to baseline, there was a mean monthly migraine frequency reduction of 46, 63 and $65 \%$, respectively, as opposed to a mean $16 \%$ reduction in the placebo group $(P=0.07$, $P=0.02, P=0.04$, respectively). The most common adverse events (in the group treated with $100 \mathrm{mg}$ a day) were as follows: paresthesia (38\%), upper respiratory tract infections $(23 \%)$, weight decrease $(15 \%)$, abdominal pain $(15 \%)$, anorexia (8\%) and somnolence $(8 \%)$. There were no dropouts in the group treated with topiramate. The incidence of adverse events was higher in the group treated with topiramate at the dosage of $200 \mathrm{mg}$, which did not appear to confer additional efficacy as opposed to the dosage of $100 \mathrm{mg}$ a day.

One class IV study [14], assessing the efficacy of topiramate for pediatric migraine, included 97 children, 75 of which were reevaluated at a first follow-up visit (after $88.7 \pm 35.7$ days) and 41 at a second follow-up visit (after $203.1 \pm 45.6$ days). Included were children reporting more than three headaches per month. Topiramate was administered at the dose of $1.4 \pm 0.74 \mathrm{mg} / \mathrm{kg} /$ day and headache frequency decreased from $16.5 \pm 10$ headaches/month to $11.6 \pm 10.2$ headaches/month $(P<0.001)$ at the first follow-up visit, with $43.1 \%$ of the patients experiencing a $50 \%$ or greater reduction in the number of headaches. By the second follow-up visit, headache frequency had decreased to $9.4 \pm 8.4$, with $56.1 \%$ of the patients experiencing a $50 \%$ or greater reduction in the number of headaches. Mean headache severity, duration and accompanying disability were also reduced. Disability was measured using the PedMIDAS score: a 50\% reduction occurred in $48.6 \%$ of the patients at the first follow-up visit and in $62.5 \%$ of patients at the second follow-up visit. One quarter of patients complained of side effects, which declined in subsequent visits and included cognitive changes $(12.5 \%)$, weight loss $(5.6 \%)$ and sensory symptoms $(2.8 \%)$. A possible bias of this study is that $50.7 \%$ of patients were on additional prophylactic medication (most often amitriptyline or divalproex).

In an open, prospective case series (class IV) study [15], topiramate was administered to 24 children with migraine, who had failed to respond to other prophylactics, at the mean dosage of $3.5 \pm 1.7 \mathrm{mg} / \mathrm{kg}$ for 4 months. Authors report a reduction in the duration and in the intensity of headaches; headache frequency reduction, however, did not reach statistical significance. Adverse events (emotional instability, paresthesia, anorexia, asthenia, weight loss) were experienced by one-third of patients. In our opinion, the baseline headache frequency $(3.6 \pm 2.7$ a month with a range of 1-12) and duration (2-6 h) did not necessarily constitute an indication for prophylactic treatment in all treated cases.

Unfortunately, the studies we reviewed were not homogeneous with regard to inclusion and exclusion criteria and with regard to outcome measures such as the "responder rate," which was not considered in all studies. Furthermore, the degree of disability caused by the migraine episodes, which is essential in the decision to 
initiate prophylactic treatment, was not assessed in all studies.

\section{Discussion}

Management of pediatric migraine can be tricky, seeing as no pharmacological treatment is formally approved or indicated in the prophylactic treatment of migraine in children and adolescents. A recent review of the pharmacological treatment options in pediatric migraine [16] found only flunarizine, which is not available in many countries, to be effective in rigorous controlled trials. Authors also concluded that there is conflicting evidence regarding propanolol and trazodone, while data from uncontrolled studies suggest cyproheptadine, amitriptyline, naproxen and antiepileptic drugs (topiramate, valproic acid and gabapentin) to be effective. Since this review was published, two randomized, placebo-controlled trials evaluating topiramate in the preventive treatment of pediatric migraine have been carried out $[10,12]$.

Taken together, data from the papers we reviewed suggest that topiramate is effective in the preventive treatment of pediatric migraine. Furthermore, topiramate dosages, which seem to be effective in the treatment of migraine in children and adolescents (2-3 mg/kg/day) are much lower than those indicated for the adjunctive treatment of epilepsy (5-9 $\mathrm{mg} / \mathrm{kg} /$ day) in children as young as 2 years.

As regards its safety and tolerability, serious adverse effects were very rare (one patient with suicidal ideation), though the risk of depression must be kept in mind. The percentage of patients experiencing side effects varied greatly among the studies, the most common ones being weight loss (which, in the RCTs [10, 12], was present in 81 and $10.2 \%$ of patients, respectively), anorexia (23.8 and $13 \%$, respectively), abdominal pain (14.3 and 10,2\%), difficulties in concentrating, somnolence/sedation (19 and $8.3 \%$ ) and paresthesia (23.8 and 8,3\%). Side effects tended to decline over time and dropout rates due to side effects were very low (range 0-6.3\%). Since these adverse events, although often transitory, may be clinically significant and distressing both for the child and its family, the decision to initiate treatment must be carefully weighed and should take the degree of disability caused by the migraine episodes into account.

An important aspect, which emerged from some of the studies, is a significant decrease in school absenteeism due to migraine [10] and a reduction in the disability caused by migraine (as assessed by PedMIDAS), leading to an improvement in the childrens' quality of life [10, 14].

In conclusion, topiramate seems to be a promising therapeutic option, though, clearly, further controlled trials are needed to confirm this data, as are studies comparing different drugs.

Conflict of interest None.

\section{References}

1. Abu-Arefeh I, Russell G (1994) Prevalence of headache and migraine in schoolchildren. Br Med J 309:765-769

2. Lipton RB, Silberstein SD, Stewart WF (1994) An update on the epidemiology of migraine. Headache 34:319-328

3. Stewart WF, Linet MS, Celentano DD, Van Natta M, Ziegler D (1991) Age and sex-specific incidence rates of migraine with and without aura. Am J Epidemiol 134:1111-1120

4. Hershey AD, Winner PK (2005) Pediatric migraine: recognition and treatment. J Am Osteopath Assoc 105(4 Suppl. 2):2S-8S

5. Lewis DW, Scott D, Rendin V (2002) Treatment of pediatric headache. Expert Opin Pharmacother 3:1433-1442

6. Stewart WF, Lipton RB, Kolodner K, Liberman J, Sawyer J (1999) Reliability of the migraine disability assessment score in a population-based sample of headache sufferers. Cephalalgia 19:107-114

7. Hershey AD, Powers SW, Vockell ALB, LeCates SL, Kabbouche MA, Maynard MK (2001) PedMIDAS: development of a questionnaire to assess disability of migraines in children. Neurology 57:2034-2039

8. Hershey AD, Powers SW, Vockell ALB, LeCates SL, Segers A, Kabbouche MA (2004) Development of a patient-based grading scale for PedMIDAS. Cephalalgia 24:844-849

9. Lewis D, Ashwal S, Hershey A, Hirtz D, Yonker M, Silberstein S; American Academy of Neurology Quality Standards Subcommittee; Practice Committee of the Child Neurology Society (2004) Practice parameter: pharmacological treatment of migraine headache in children and adolescents: report of the American Academy of Neurology Quality Standards Subcommittee and the Practice Committee of the Child Neurology Society. Neurology 28(63):2215-2224

10. Lakshmi CV, Singhi P, Malhi P, Ray MJ (2007) Topiramate in the prophylaxis of pediatric migraine: a double-blind placebocontrolled trial. Child Neurol 22(7):829-835

11. Classification Committee for the International Headache Society (2004) The International classification of headache disorders. Cephalalgia 24:1-160

12. Winner P, Pearlmann EM, Linder SL, Jordan DM, Fisher AC, Hulihan J (2005) Topiramate for migraine prevention in children: a randomized, double-blind, placebo-controlled trial. Headache 45:1304-1312

13. Winner P, Gendolla A, Stayer C, Wang S, Yuen E, Battisti W, Nye JS (2006) Topiramate for migraine prevention in adolescents: a pooled analysis of efficacy and safety. Headache 46:1503-1510

14. Hershey AD, Powers SW, Vockell AL, LeCates S, Kabbouche M (2002) Effectiveness of topiramate in the prevention of childhood headaches. Headache 42:810-818

15. Campistol J, Campos J, Casas C, Herranz JL (2005) Topiramate in the prophylactic treatment of migraine in children. J Child Neurol 20:251-253

16. Lewis DW, Winner P (2006) The pharmacological treatment options for pediatric migraine: an evidence-based appraisal. NeuroRx 3(2):181-191 